\title{
Effect of Polyamine on Physio-Biochemical Traits in Sugarcane Grown under Sodic Soil
}

\author{
P. S. Chougule*, J. K. Kharat, A. A. Kale, R. M. Naik, A. S. Jadhav and N. S. Kute \\ Post Graduate Institute, Mahatma Phule Krishi Vidyapeeth, Rahuri, \\ Ahmednagar (M.S.) - 413722, India \\ *Corresponding author
}

\section{A B S T R A C T}

\begin{tabular}{|l|}
\hline Ke y w o r d s \\
Sugarcane, \\
Polyamines, Sodic \\
soil, Morphological \\
traits, Physio- \\
biochemical traits
\end{tabular}

\section{Introduction}

Saccharum has been derived from Sanskrit word Sarkara meaning sugar. Sugarcane is tropical and subtropical world crop, which is propagated through sets. Sugarcane (Saccharum officinarum) is one of the most important commercial crops in the world. India is the second position in area, production and productivity in the world next to Brazil. Globally in world area during 2016-2017was 20.42 million ha, production - 1333 million tons and Productivity - 65.2 tons/ha respectively. In India Area - 4.608 million ha, production - 289.6 million tons and productivity - 62.2 tons/ha. In India total area of cultivation of sugarcane is 4.38 Mha, production is about $306.72 \mathrm{MT}$ and average 
yield of sugarcane is 69.88 ton ha $\mathrm{a}^{-1}$ during the year 2016-17. In Maharashtra the total area cultivation of sugarcane is 0.63 Mha and which is about 14.42 percent of all India sugarcane cultivation. The production of sugarcane in Maharashtra is 50.64 MT which is about 16.31 percent of all India production. The average yield of sugarcane is 80 ton $\mathrm{ha}^{-1}$ (Anonymous, 2018).

Maintaining crop yields under adverse environmental stresses is probably the major challenge facing modern agriculture. Due to the excessive irrigation the soils in the Maharashtra have become saline. Numerous attempts have been made to improve the salinity tolerance of a variety of crops by traditional breeding programs, but the progress to develop such salt-resistant plants is very slow and commercial success is limited (Duan et al., 2008). Sugarcane is a glycophyte, with its yield falling to $50 \%$ or even more of its true potential which could possibly be due to the accumulation of toxic ions. Being highly crossbred, sugarcane exhibits a significant genetic variability in nature (Wahid et al., 1997).

Salinization is one of the most devastating forms of land degradation threatening food production worldwide, especially in arid and semi-arid countries. However, climate change predictions indicated less rainfall and higher temperatures in the future in most of the agricultural regions. So, experts worry that the changes will lead to even more saline lands and predict that salinity will be increased from 4 to $9 \mathrm{dSm}^{-1}$ in the future. Progress in developing salt tolerant varieties has been very slow because of less knowledge on the mechanism of salt damage and complex nature of salt tolerance. Thus, understanding the adaptive mechanisms of each crop becomes necessary to improve or produce the salt resistant genotypes. Salinity may cause damage to the plants through osmotic stress, nutrient imbalance and specific ion toxicity (Munns et al., 1986).

Polyamines (PAs), including the spermidine, spermine and putrescine are now regarded as plant growth regulators and secondary messenger in signaling pathways (Kusano et al., 2008). Because of their cationic nature at physiological $\mathrm{pH}$, PAs are able to interact with proteins, nucleic acids, membrane phospholipids and cell wall constituents, there by stabilizing these molecules. PAs have been reported to be involved in defense response to biotic and abiotic stresses (Alcázar et al., 2010). Exogenously applied PAs have been reported to substantially enhance salt tolerance in rice plants (Chattopadhyay et al., 2002).

During the past few years, a number of genes encoding PA biosynthetic enzymes have been isolated in different plants. Numerous transgenic plants with perturbed PA metabolism have been produced to elucidate their cellular functions furthermore, the updated transcriptomic and proteomic approaches have been employed to PA function research; however, the precise roles of PAs remain largely elusive. With the recent reestablishment of the roles of reactive oxygen species (ROS) in the stress response and the relationship between the nitric oxide biosynthesis and PA titers, the roles of PA in the stress response are attracting more attention than ever, which provides a good chance to make a retrospect to the past studies on the relationships between PAs and stresses, including biotic and abiotic stresses (Pang et al., 2007).

Polyamines (PAs) are ubiquitous lowmolecular-weight aliphatic amines that are involved in regulation of plant growth and development. PAs are also implicated in a wide range of environmental stress tolerance in plants. New roles are being discovered every day for these interesting molecules in 
the plant world. In higher plants, the most common PAs are spermidine (Spd) their diamine obligate precursor putrescine (Put). Like PAs displaying high biological activity are involved in a wide array of fundamental processes in plants, such as replication and gene expression, growth and development, senescence, membrane stabilization, enzyme activity modulation and adaptation to abiotic stresses (Alet et al., 2012). However, the precise physiological function and mechanism of action of PAs still remain unclear. In contrast to the reliable works on the role of PAs in plants defense against biotic and abiotic stresses, few reports recently indicated that PAs may act as cellular signals in intrinsic talk with hormonal pathways including ABA (Alcazar et al., 2010; Gill and Tuteja, 2010). Changes in plant PA metabolism occur in response to a variety of abiotic stresses (Alcazar et al., 2006; Gill and Tuteja, 2010). These changes in cellular PA under stress only provide clues on its possible implication in stress response, but they do not provide evidence of its role in counteracting stress. Hence, to understand whether PA actually protect cells from stress induced damages, exogenous application of PA, which is expected to increase endogenous PA, has been investigated before or during stress (Wang et al., 2007). It has been reported that exogenous application of PAs could alleviate salt induced reduction in photosynthetic efficiency, but this effect is strongly dependent both on PAs concentration or types and stress levels (Duan et al., 2008). The result obtained by Zhang et al., (2009) suggested that Put strongly affects photosynthetic apparatus involving in enhancement of photochemical quenching rather than regulation of stomatal closure or opening. Several publications have reported that changes of endogenous PA level and forms are involved in regulating the photochemical efficiency of salt-stressed plants, and PAs metabolism-related enzymes are closely correlated with photosynthesis.
Therefore overall aim of this study is to elucidate the polyamine induced physiological and biochemical changes responsible for induction of sodicity tolerance in sugarcane.

\section{Materials and Methods}

\section{Experimental details}

The sugarcane sets of a salt tolerant viz., CoM 0265 and a salt susceptible CoC 671 were obtained from CSRS Padegaon. The sets with an eye bud were washed and sterilized with sodium hypochlorite $(0.1 \% \mathrm{w} / \mathrm{v})$. The sets were dipped in the solution of individual polyamines viz., 0,100 and $500 \mu \mathrm{M}$ of putrescine and spermidine. These setts were planted in sodic soil and normal soil in triplicate. The two foliar applications of these polyamines were carried out 45 days after planting with 8 days interval. The leaf samples were collected after 65 days after planting for the biochemical analyses.

\section{Chlorophyll a and b content and Total chlorophyll, by Arnon, (1949)}

The leaf samples of sugarcane were cut into small pieces and known weight $(0.2 \mathrm{~g})$ of fresh leaf sample was macerated in a mortar and pestle and extracted with $20 \mathrm{~mL}$ of 80 per cent acetone. The content was centrifuged at $5000 \mathrm{xg}$ for $10 \mathrm{~min}$ and the supernatant was collected. The final volume of extract was made to $50 \mathrm{~mL}$. The extinction of chlorophyll extract was recorded at 645 and $663 \mathrm{~nm}$ on a Spectrophotometer and a blank was run with 80 per cent acetone.

\section{Lipid peroxidation by Heath and Packer, (1968)}

Sample extraction: Leaf sample $0.2 \mathrm{~g}$ was homogenized in $4 \mathrm{~mL}$ of $0.1 \%$ TCA. The homogenate was centrifuged at $15000 \mathrm{~g}$ for 15 min and the supernatant was used for the 
estimation of TBARS content. Four $\mathrm{mL}$ of 0.5 $\%$ TBA in $20 \%$ TCA was added in $10 \mathrm{~mL}$ aliquot of the supernatant. The mixture was heated at $95^{\circ} \mathrm{C}$ for $30 \mathrm{~min}$ in the lab electric oven and then cooled in an ice bath.

After cooling the aliquot was centrifuged at $10,000 \mathrm{Xg}$ for $10 \mathrm{~min}$. The absorbance of the clear supernatant was recorded at $532 \mathrm{~nm}$. Values of nonspecific absorption recorded at $600 \mathrm{~nm}$ and were subtracted from the values recorded at $532 \mathrm{~nm}$. The TBARS content was calculated by using extinction coefficient $\mathrm{E}=$ 155 nmoles of MDA formed $\mathrm{mg}^{-1}$ fr. wt.

\section{Reducing sugars by Nelson-Somogyi (1944)}

$0.2 \mathrm{~mL}$ of sugar extract and $0.8 \mathrm{~mL}$ of distilled water, one $\mathrm{mL}$ of copper reagent was added. The content were mixed and heated for 20 min. in boiling water bath. The tubes were cooled. One $\mathrm{mL}$ of arsenomolybdate reagent was added and mixed well. This mixture was diluted with $7 \mathrm{~mL}$ of distilled water and the absorbance was taken at $520 \mathrm{~nm}$ against blank.

The reducing were calculated from a standard curve for D-glucose and expressed as milligrams per $100 \mathrm{~g}$ dry weight.

\section{Total sugars by Nelson-Somogyi (1944)}

To the $25 \mathrm{~mL}$ of ethanolic extract $2.5 \mathrm{~mL}$ of hydrochloric acid was added and kept for 24 hours for inversion. It was neutralized with $0.1 \mathrm{~N}$ sodium hydroxide using $\mathrm{pH}$ meter and the volume was made to $50 \mathrm{ml}$. The hydrolyzed sugars were estimated as total sugars from the leaves of sugarcane grown in normal and sodic soil.

\section{Non-Reducing sugars by Nelson-Somogyi (1944)}

Non-reducing sugars content were calculated as difference between total and reducing sugars.

\section{Results and Discussion}

\section{Chlorophyll ' $a$ '}

Chlorophyll 'a' content was decreased in CoC 671 a salt susceptible cultivar and CoM 0265 a salt tolerant cultivar when grown in sodic soil (Table 1). However, with the application of polyamines chlorophyll 'a' of sugarcane grown under normal and sodic soil was increased with increase in the polyamine concentration in both the cultivar (Table 1). The chlorophyll 'a' of the variety CoM 0265 by the application of putrescine and spermidine was $0.76 \mathrm{mg} \mathrm{g}^{-1}(0 \mu \mathrm{M}), 0.91$ $\mathrm{mg} \mathrm{g}^{-1}(100 \mu \mathrm{M}), 1.03 \mathrm{mg} \mathrm{g}^{-1}(500 \mu \mathrm{M})$ and $0.74 \mathrm{mg} \mathrm{g}^{-1}(0 \mu \mathrm{M}), 0.87 \mathrm{mg} \mathrm{g}^{-1}(100 \mu \mathrm{M}), 0.97$ $\mathrm{mg} \mathrm{g}^{-1}(500 \mu \mathrm{M})$ respectively in normal soil. The chlorophyll 'a' of the variety CoM 0265 with the application of putrescine and spermidine was $0.49 \mathrm{mg} \mathrm{g}^{-1}(0 \mu \mathrm{M}), 1.20 \mathrm{mg} \mathrm{g}^{-}$ ${ }^{1}(100 \mu \mathrm{M}), 1.97 \mathrm{mg} \mathrm{g}^{-1}(500 \mu \mathrm{M})$ and $0.47 \mathrm{mg}$ $\mathrm{g}^{-1}(0 \mu \mathrm{M}), 0.96 \mathrm{mg} \mathrm{g}^{-1}(100 \mu \mathrm{M}), 1.49 \mathrm{mg} \mathrm{g}^{-}$ ${ }^{1}(500 \mu \mathrm{M})$ respectively in sodic soil. The chlorophyll ' $a$ ' of the variety $\mathrm{CoC} 671$ by the application of putrescine and spermidine was $0.72 \mathrm{mg} \mathrm{g}^{-1}(0 \mu \mathrm{M}), 0.89 \mathrm{mg} \mathrm{g}^{-1}(100 \mu \mathrm{M}), 1.03$ $\mathrm{mg} \mathrm{g}^{-1}(500 \mu \mathrm{M})$ and $0.70 \mathrm{mg} \mathrm{g}^{-1}(0 \mu \mathrm{M})$, $0.85 \mathrm{mg} \mathrm{g}^{-1}(100 \mu \mathrm{M}), 0.99 \mathrm{mg} \mathrm{g}^{-1}(500 \mu \mathrm{M})$ respectively in normal soil.

The chlorophyll ' $\mathrm{a}$ ' of the variety $\mathrm{CoC} 671$ by the application of putrescine and spermidine was $0.41 \mathrm{mg} \mathrm{g}^{-1}(0 \mu \mathrm{M}), 1.23 \mathrm{mg} \mathrm{g}^{-1}(100 \mu \mathrm{M})$, $1.94 \mathrm{mg} \mathrm{g}^{-1}(500 \mu \mathrm{M})$ and $0.43 \mathrm{mg} \mathrm{g}^{-1}(0 \mu \mathrm{M})$, $1.16 \mathrm{mg} \mathrm{g}^{-1}(100 \mu \mathrm{M}), 1.85 \mathrm{mg} \mathrm{g}^{-1}(500 \mu \mathrm{M})$ respectively in sodic soil.

\section{Chlorophyll ' $b$ '}

From Table 2 it was noticed that chlorophyll ' $b$ ' in sugarcane varieties CoM 0265 salt tolerant and $\mathrm{CoC} 671$ salt susceptible was reduced when grown in sodic soil. Effect of application of polyamine on chlorophyll ' $b$ ' of sugarcane grown under normal and sodic soil revealed that there was increase in chlorophyll 
' $b$ ' with increase in the polyamine concentration under normal and sodic soil in both on the cultivar (Table 2). The chlorophyll ' $b$ ' of the variety CoM 0265 by the application of putrescine and spermidine was $0.54 \mathrm{mg} \mathrm{g}^{-1}$ $(0 \mu \mathrm{M}), 0.69 \mathrm{mg} \mathrm{g}^{-1}(100 \mu \mathrm{M}), 0.81 \mathrm{mg} \mathrm{g}^{-1}$ $(500 \mu \mathrm{M})$ and $0.74 \mathrm{mg} \mathrm{g}^{-1}(0 \mu \mathrm{M}), 0.87 \mathrm{mg} \mathrm{g}^{-1}$ $(100 \mu \mathrm{M}), 0.97 \mathrm{mg} \mathrm{g}^{-1}(500 \mu \mathrm{M})$ respectively in normal soil.

The chlorophyll ' $b$ ' of the variety CoM 0265 by the application of putrescine and spermidine was $0.47 \mathrm{mg} \mathrm{g}^{-1}(0 \mu \mathrm{M}), 1.18$

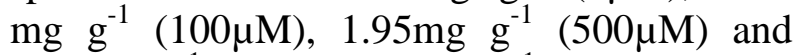
$0.47 \mathrm{mg} \mathrm{g}^{-1}(0 \mu \mathrm{M}), 0.96 \mathrm{mg} \mathrm{g}^{-1}(100 \mu \mathrm{M}), 1.49$ $\mathrm{mg} \mathrm{g}^{-1}(500 \mu \mathrm{M})$ respectively in sodic soil. The chlorophyll ' $b$ ' of the variety $\mathrm{CoC} 671$ by the application of putrescine and spermidine was $0.46 \mathrm{mg} \mathrm{g}^{-1}(0 \mu \mathrm{M}), 0.63 \mathrm{mg} \mathrm{g}^{-1}(100 \mu \mathrm{M}), 0.77$ $\mathrm{mg} \mathrm{g}^{-1}(500 \mu \mathrm{M})$ and $0.46 \mathrm{mg} \mathrm{g}^{-1}(0 \mu \mathrm{M})$, $0.61 \mathrm{mg} \mathrm{g}^{-1}(100 \mu \mathrm{M}), 0.75 \mathrm{mg} \mathrm{g}^{-1}(500 \mu \mathrm{M})$ respectively in normal soil. The chlorophyll 'b' of the variety $\mathrm{CoC} 671$ by the application of putrescine and spermidine was $0.41 \mathrm{mg} \mathrm{g}^{-1}$ $(0 \mu \mathrm{M}), \quad 1.23 \mathrm{mg} \mathrm{g}^{-1}(100 \mu \mathrm{M}), \quad 1.94 \mathrm{mg} \mathrm{g}^{-1}$ $(500 \mu \mathrm{M})$ and $0.43 \mathrm{mg} \mathrm{g}^{-1}(0 \mu \mathrm{M}), 1.16 \mathrm{mg} \mathrm{g}^{-}$ ${ }^{1}(100 \mu \mathrm{M}), 1.85 \mathrm{mg} \mathrm{g}^{-1}(500 \mu \mathrm{M})$ in sodic soil.

\section{Total chlorophyll}

The total chlorophyll in leaves of sugarcane varieties viz. CoM 0265 a salt tolerant and $\mathrm{CoC} 671$ a salt susceptible were decreased when planted in sodic soil (Table 3). However, the application of polyamine on total chlorophyll of sugarcane grown under normal and sodic soil the revealed that it was increased is decreased with increase in the polyamine concentrations content in both on the cultivar (Table 3). The total chlorophyll content in the variety COM 265 by the application of putrescine and spermidine was $1.30 \mathrm{mg} \mathrm{g}^{-1}(0 \mu \mathrm{M}), 1.60 \mathrm{mg} \mathrm{g}^{-1}(100 \mu \mathrm{M}), 1.84$ $\mathrm{mg} \mathrm{\textrm {g } ^ { - 1 }}(500 \mu \mathrm{M})$ and $1.26 \mathrm{mg} \mathrm{g}^{-1}(0 \mu \mathrm{M})$, $1.52 \mathrm{mg} \mathrm{g}^{-1}(100 \mu \mathrm{M}), 1.72 \mathrm{mg} \mathrm{g}^{-1}(500 \mu \mathrm{M})$ respectively in normal soil.

\section{Lipid peroxidation}

Lipid peroxidation was reduced in the sugarcane varieties viz., CoM 0265, salt tolerant and $\mathrm{CoC}$ 671, salt susceptible grown in sodic soil when treated with polyamines (Table 4). Effect of polyamine on lipid peroxidation of sugarcane varieties grown under normal and sodic soil revealed that with increase in the polyamine concentration under normal and sodic soil in both on the cultivar there was reduction of lipid peroxidation rate (Table 4). The lipid peroxiation of the variety CoM 0265 at concentration of putrescine and spermidine was $28.03 \eta$ moles of MDA formed $\mathrm{mg}^{-1}$ fr.wt. $(0 \mu \mathrm{M}), 28.00$ ๆmoles of MDA formed $\mathrm{mg}^{-1}$ fr.wt. $(100 \mu \mathrm{M})$, 27.99 nmoles of MDA formed $\mathrm{mg}^{-1}$ fr.wt. $(500 \mu \mathrm{M})$ and $28.00 \eta$ moles of MDA formed $\mathrm{mg}^{-1}$ fr.wt. $(0 \mu \mathrm{M}), 28.16 \eta$ moles of MDA formed $\mathrm{mg}^{-1}$ fr.wt. $(100 \mu \mathrm{M}), 28.17 \eta$ moles of MDA formed $\mathrm{mg}^{-1}$ fr.wt. $(500 \mu \mathrm{M})$ in normal soil.

The lipid peroxidation of the variety $\mathrm{CoM}$ 0265 at concentration of putrescine and spermidine was $28.33 \eta$ moles of MDA formed $\mathrm{mg}^{-1}$ fr.wt. $(0 \mu \mathrm{M}), 28.19 \eta$ moles of MDA formed $\mathrm{mg}^{-1}$ fr.wt. $(100 \mu \mathrm{M}), 28.10 \eta$ moles of MDA formed $\mathrm{mg}^{-1}$ fr.wt. $(500 \mu \mathrm{M})$ and $28.31 \eta$ moles of MDA formed $\mathrm{mg}^{-1}$ fr.wt. $(0 \mu \mathrm{M}), 28.21 \eta$ moles of MDA formed $\mathrm{mg}^{-1}$ fr.wt. $(100 \mu \mathrm{M}), 28.16 \eta$ moles of MDA formed $\mathrm{mg}^{-1}$ fr.wt. $(500 \mu \mathrm{M})$ in sodic soil.

The lipid peroxidation of the variety $\mathrm{CoC} 671$ at concentration of putrescine and spermidine was $18.55 \eta$ moles of MDA formed $\mathrm{mg}^{-1}$ fr.wt. $(0 \mu \mathrm{M}), 18.50 \eta$ moles of MDA formed $\mathrm{mg}^{-1}$ fr.wt. $(100 \mu \mathrm{M}), 18.48 \eta$ moles of MDA formed $\mathrm{mg}^{-1}$ fr.wt. $(500 \mu \mathrm{M})$ and $18.41 \eta$ moles of MDA formed $\mathrm{mg}^{-1}$ fr.wt. $(0 \mu \mathrm{M}), 18.40$ moles of MDA formed $\mathrm{mg}^{-1}$ fr.wt. $(100 \mu \mathrm{M})$, $18.38 \eta$ moles of MDA formed $\mathrm{mg}^{-1}$ fr.wt. $(500 \mu \mathrm{M})$ in normal soil. 
Table.1 Effect of polyamines on chlorophyll a in sugarcane leaves grown on normal and sodic soils

\begin{tabular}{|c|c|c|c|c|c|c|c|c|}
\hline \multirow{4}{*}{$\begin{array}{l}\text { Sr. } \\
\text { No. }\end{array}$} & \multirow{4}{*}{$\begin{array}{l}\text { Name of the } \\
\text { cultivar/ } \\
\text { Name of the } \\
\text { polyamine }\end{array}$} & \multicolumn{6}{|c|}{ Chlorophyll a (mg g $\left.{ }^{-1}\right)$} & \multirow{4}{*}{$\begin{array}{c}\text { Fold Increase }(+) / \\
\text { Fold Decrease } \\
(-)\end{array}$} \\
\hline & & \multicolumn{3}{|c|}{ Normal soil } & \multirow{2}{*}{\multicolumn{3}{|c|}{ Sodic soil }} & \\
\hline & & \multicolumn{3}{|c|}{ Concentration of the polyamine $(\mu \mathrm{M})$} & & & & \\
\hline & & $\mathbf{0}$ & 100 & 500 & 0 & 100 & 500 & \\
\hline 1. & \multicolumn{7}{|c|}{ CoM 265 (Salt tolerant cultivar) } & \\
\hline \multirow[t]{3}{*}{ a. } & \multirow[t]{3}{*}{ Putrescine } & $\begin{array}{l}0.76 \\
(00)\end{array}$ & $\begin{array}{c}1.20 \\
(+1.58)\end{array}$ & $\begin{array}{c}1.97 \\
(+2.59)\end{array}$ & $\begin{array}{c}0.49 \\
(-0.64)\end{array}$ & $\begin{array}{c}0.91 \\
(+1.19)\end{array}$ & $\begin{array}{c}1.03 \\
(+1.35)\end{array}$ & $\begin{array}{l}\text { Over the control of } \\
\text { normal soil }\end{array}$ \\
\hline & & - & - & - & $(00)$ & $(+1.88)$ & $(+2.10)$ & $\begin{array}{l}\text { Over the control of } \\
\text { sodic soil }\end{array}$ \\
\hline & & - & - & - & - & $(+0.76)$ & $(-0.52)$ & $\begin{array}{l}\text { Over respective } \\
\text { Putrescine conc. }\end{array}$ \\
\hline \multirow[t]{3}{*}{ b. } & \multirow[t]{3}{*}{ Spermidine } & $\begin{array}{l}0.74 \\
(00)\end{array}$ & $\begin{array}{c}0.96 \\
(+1.30)\end{array}$ & $\begin{array}{c}1.49 \\
(+2.01)\end{array}$ & $\begin{array}{c}0.47 \\
(-0.63)\end{array}$ & $\begin{array}{c}0.87 \\
(+1.18)\end{array}$ & $\begin{array}{c}0.97 \\
(+1.31)\end{array}$ & $\begin{array}{l}\text { Over the control of } \\
\text { normal soil }\end{array}$ \\
\hline & & - & - & - & $(00)$ & $(+1.85)$ & $(+2.06)$ & $\begin{array}{l}\text { Over the control of } \\
\text { sodic soil }\end{array}$ \\
\hline & & - & - & - & - & $(-0.97)$ & $(+0.65)$ & $\begin{array}{l}\text { Over respective } \\
\text { Spermidine conc }\end{array}$ \\
\hline 2. & \multicolumn{7}{|c|}{ CoC 671 (Salt susceptible cultivar) } & \\
\hline \multirow[t]{3}{*}{ a. } & \multirow[t]{3}{*}{ Putrescine } & $\begin{array}{l}0.72 \\
(00)\end{array}$ & $\begin{array}{c}1.23 \\
(+1.71)\end{array}$ & $\begin{array}{c}1.94 \\
(+2.70)\end{array}$ & $\begin{array}{c}0.41 \\
(-0.57)\end{array}$ & $\begin{array}{c}0.89 \\
(+1.24)\end{array}$ & $\begin{array}{c}1.03 \\
(+1.43)\end{array}$ & $\begin{array}{l}\text { Over the control of } \\
\text { normal soil }\end{array}$ \\
\hline & & - & - & - & $(00)$ & $(+2.17)$ & $(+2.51)$ & $\begin{array}{l}\text { Over the control of } \\
\text { sodic soil }\end{array}$ \\
\hline & & - & - & - & - & $(-0.72)$ & $(-0.53)$ & $\begin{array}{l}\text { Over respective } \\
\text { Putrescine conc. }\end{array}$ \\
\hline \multirow[t]{11}{*}{ b. } & \multirow[t]{3}{*}{ Spermidine } & $\begin{array}{l}0.70 \\
(00)\end{array}$ & $\begin{array}{c}1.16 \\
(+1.66)\end{array}$ & $\begin{array}{c}1.85 \\
(+2.64)\end{array}$ & $\begin{array}{c}0.43 \\
(-0.62)\end{array}$ & $\begin{array}{c}0.85 \\
(+1.22)\end{array}$ & $\begin{array}{c}0.99 \\
(+1.42)\end{array}$ & $\begin{array}{l}\text { Over the control of } \\
\text { normal soil }\end{array}$ \\
\hline & & - & - & - & (00) & $(+1.98)$ & $(+2.30)$ & $\begin{array}{l}\text { Over the control of } \\
\text { sodic soil }\end{array}$ \\
\hline & & - & - & - & - & $(-0.73)$ & $(-0.54)$ & $\begin{array}{l}\text { Over respective } \\
\text { Spermidine conc }\end{array}$ \\
\hline & Comparison & $\begin{array}{l}\text { S.Em. } \\
\pm\end{array}$ & $\begin{array}{l}\mathrm{CD} \text { at } \\
5 \%\end{array}$ & & Comparison & $\begin{array}{l}\text { S.Em. } \\
\pm\end{array}$ & $\begin{array}{l}\mathrm{CD} \text { at } \\
5 \%\end{array}$ & \\
\hline & Variety (V) & 0.004 & 0.012 & & $\mathrm{~T} * \mathrm{~L}$ & 0.007 & 0.021 & \\
\hline & Soil (S) & 0.004 & 0.012 & & $\mathrm{~T} * \mathrm{~S}$ & 0.006 & 0.017 & \\
\hline & $\begin{array}{c}\text { Type of } \\
\text { polyamine }(\mathrm{T})\end{array}$ & 0.004 & 0.012 & & $\mathrm{~V} * \mathrm{~S} * \mathrm{~T}$ & 0.008 & 0.025 & \\
\hline & $\begin{array}{c}\text { Conc. of } \\
\text { Polyamines (L) }\end{array}$ & 0.005 & 0.015 & & $\mathrm{~V} * \mathrm{~T} * \mathrm{~L}$ & 0.010 & 0.030 & \\
\hline & $\mathrm{V} * \mathrm{~S}$ & 0.006 & 0.017 & & $\mathrm{~S} * \mathrm{~T} * \mathrm{~L}$ & 0.010 & 0.030 & \\
\hline & $\mathrm{V} * \mathrm{~T}$ & 0.006 & 0.017 & & $\mathrm{~V} * \mathrm{~S} * \mathrm{~L}$ & 0.010 & 0.030 & \\
\hline & & & & & $\mathbf{V} * \mathbf{S} * \mathbf{T} * \mathbf{L}$ & 0.015 & 0.043 & \\
\hline
\end{tabular}


Table.2 Effect of polyamines on chlorophyll $\mathrm{b}$ in sugarcane leaves grown on normal and sodic soils

\begin{tabular}{|c|c|c|c|c|c|c|c|c|}
\hline \multirow{4}{*}{$\begin{array}{l}\text { Sr. } \\
\text { No. }\end{array}$} & \multirow{4}{*}{$\begin{array}{l}\text { Name of the } \\
\text { cultivar/ } \\
\text { Name of the } \\
\text { polyamine }\end{array}$} & \multicolumn{6}{|c|}{ Chlorophyll b (mg g $\left.{ }^{-1}\right)$} & \multirow{4}{*}{$\begin{array}{c}\text { Fold Increase }(+) / \\
\text { Fold Decrease } \\
(-)\end{array}$} \\
\hline & & \multicolumn{3}{|c|}{ Normal soil } & \multirow{2}{*}{\multicolumn{3}{|c|}{$\begin{array}{r}\text { Sodic soil } \\
\text { f the polyamine }(\mu \mathrm{M})\end{array}$}} & \\
\hline & & & & ntration & & & & \\
\hline & & $\mathbf{0}$ & 100 & 500 & 0 & 100 & 500 & \\
\hline 1. & \multicolumn{7}{|c|}{ CoM 265 (Salt tolerant cultivar) } & \\
\hline a. & Putrescine & $\begin{array}{l}0.54 \\
(00) \\
-\end{array}$ & $\begin{array}{c}0.69 \\
(+1.28) \\
- \\
-\end{array}$ & $\begin{array}{c}0.81 \\
(+1.5) \\
- \\
-\end{array}$ & $\begin{array}{c}0.47 \\
(-0.87) \\
(00) \\
-\end{array}$ & $\begin{array}{c}1.18 \\
(+2.18) \\
(+2.51) \\
(+1.71)\end{array}$ & $\begin{array}{c}1.95 \\
(+3.61) \\
(+4.15) \\
(+2.41)\end{array}$ & $\begin{array}{l}\text { Over the control of } \\
\text { normal soil } \\
\text { Over the control of } \\
\text { sodic soil } \\
\text { Over respective } \\
\text { Putrescine conc. }\end{array}$ \\
\hline b. & Spermidine & $\begin{array}{c}0.74 \\
(00) \\
-\end{array}$ & $\begin{array}{c}0.87 \\
(+1.17) \\
-\end{array}$ & $\begin{array}{c}0.97 \\
(+1.31) \\
-\end{array}$ & $\begin{array}{c}0.47 \\
(-0.63) \\
(00) \\
-\end{array}$ & $\begin{array}{c}0.96 \\
(+1.30) \\
(+2.04) \\
(+1.10)\end{array}$ & $\begin{array}{c}1.49 \\
(+2.01) \\
(+3.17) \\
(+1.54)\end{array}$ & $\begin{array}{l}\text { Over the control of } \\
\text { normal soil } \\
\text { Over the control of } \\
\text { sodic soil } \\
\text { Over respective } \\
\text { Spermidine conc } \\
\end{array}$ \\
\hline 2. & \multicolumn{7}{|c|}{ CoC 671 (Salt susceptible cultivar) } & \\
\hline a. & Putrescine & $\begin{array}{c}0.46 \\
(00) \\
-\end{array}$ & $\begin{array}{c}0.63 \\
(+1.37) \\
-\end{array}$ & $\begin{array}{c}0.77 \\
(+1.67) \\
- \\
-\end{array}$ & $\begin{array}{c}0.41 \\
(-0.89) \\
(00) \\
-\end{array}$ & $\begin{array}{c}1.23 \\
(+2.67) \\
(+3) \\
(+1.95)\end{array}$ & $\begin{array}{c}1.94 \\
(+4.21) \\
(+4.73) \\
(+2.51)\end{array}$ & $\begin{array}{l}\text { Over the control of } \\
\text { normal soil } \\
\text { Over the control of } \\
\text { sodic soil } \\
\text { Over respective } \\
\text { Putrescine conc. }\end{array}$ \\
\hline \multirow[t]{9}{*}{ b. } & Spermidine & $\begin{array}{c}0.46 \\
(00) \\
-\end{array}$ & $\begin{array}{c}0.61 \\
(+1.32) \\
-\end{array}$ & $\begin{array}{c}0.75 \\
(+1.63) \\
-\end{array}$ & $\begin{array}{c}0.43 \\
(-0.93) \\
(00) \\
-\end{array}$ & $\begin{array}{c}1.16 \\
(+2.52) \\
(+2.69) \\
(+1.90)\end{array}$ & $\begin{array}{c}1.85 \\
(+4.02) \\
(+4.30) \\
(+2.46)\end{array}$ & $\begin{array}{l}\begin{array}{l}\text { Over the control of } \\
\text { normal soil }\end{array} \\
\text { Over the control of } \\
\text { sodic soil } \\
\text { Over respective } \\
\text { Spermidine conc }\end{array}$ \\
\hline & Comparison & $\begin{array}{l}\text { S.Em. } \\
\pm\end{array}$ & $\begin{array}{l}\mathrm{CD} \text { at } \\
5 \%\end{array}$ & & Comparison & $\begin{array}{l}\text { S.Em. } \\
\pm\end{array}$ & $\begin{array}{l}\mathrm{CD} \text { at } \\
5 \%\end{array}$ & \\
\hline & Variety (V) & 0.005 & 0.014 & & $\mathrm{~T} * \mathrm{~L}$ & 0.008 & 0.024 & \\
\hline & Soil (S) & 0.005 & 0.014 & & $\mathrm{~T} * \mathrm{~S}$ & 0.007 & 0.019 & \\
\hline & $\begin{array}{c}\text { Type of } \\
\text { polyamine }(\mathrm{T})\end{array}$ & 0.005 & 0.014 & & $\mathrm{~V} * \mathrm{~S} * \mathrm{~T}$ & 0.009 & 0.028 & \\
\hline & $\begin{array}{c}\text { Conc. of } \\
\text { Polyamines (L) }\end{array}$ & 0.006 & 0.017 & & $\mathrm{~V} * \mathrm{~T} * \mathrm{~L}$ & 0.012 & 0.034 & \\
\hline & $\mathrm{V} * \mathrm{~S}$ & 0.007 & 0.019 & & $\mathrm{~S} * \mathrm{~T} * \mathrm{~L}$ & 0.012 & 0.034 & \\
\hline & $\mathrm{V} * \mathrm{~T}$ & 0.007 & 0.019 & & $\mathrm{~V} * \mathrm{~S} * \mathrm{~L}$ & 0.012 & 0.034 & \\
\hline & & & & & $\mathbf{V} * \mathbf{S} * \mathbf{T} * \mathbf{L}$ & 0.017 & 0.048 & \\
\hline
\end{tabular}


Table.3 Effect of polyamines on total chlorophyll in sugarcane leaves grown on normal and sodic soils

\begin{tabular}{|c|c|c|c|c|c|c|c|c|}
\hline \multirow{4}{*}{$\begin{array}{l}\text { Sr. } \\
\text { No }\end{array}$} & \multirow{4}{*}{$\begin{array}{l}\text { Name of the } \\
\text { cultivar/ } \\
\text { Name of the } \\
\text { polyamine }\end{array}$} & \multicolumn{6}{|c|}{ Total Chlorophyll $\left(\mathrm{mg} \mathrm{g}^{-1}\right)$} & \multirow{4}{*}{$\begin{array}{c}\text { Fold Increase }(+) / \\
\text { Fold Decrease } \\
(-)\end{array}$} \\
\hline & & \multicolumn{3}{|c|}{ Normal soil } & \multirow{2}{*}{\multicolumn{3}{|c|}{ Sodic soil }} & \\
\hline & & \multicolumn{3}{|c|}{ Concentration of the polyamine $(\mu \mathrm{M})$} & & & & \\
\hline & & 0 & 100 & 500 & 0 & 100 & 500 & \\
\hline 1. & \multicolumn{7}{|c|}{ CoM 265 (Salt tolerant cultivar) } & \\
\hline \multirow[t]{3}{*}{ a. } & \multirow[t]{3}{*}{ Putrescine } & $\begin{array}{l}1.30 \\
(00)\end{array}$ & $\begin{array}{c}1.60 \\
(+1.23)\end{array}$ & $\begin{array}{c}1.84 \\
(+1.42)\end{array}$ & $\begin{array}{c}0.96 \\
(-0.73)\end{array}$ & $\begin{array}{c}2.38 \\
(+1.83)\end{array}$ & $\begin{array}{c}3.92 \\
(+3.02)\end{array}$ & $\begin{array}{l}\text { Over the control of } \\
\text { normal soil }\end{array}$ \\
\hline & & - & - & - & $(00)$ & $(+2.47)$ & $(+4.08)$ & $\begin{array}{l}\text { Over the control of } \\
\text { sodic soil }\end{array}$ \\
\hline & & - & - & - & - & $(+1.48)$ & $(+2.13)$ & $\begin{array}{l}\text { Over respective } \\
\text { Putrescine conc. }\end{array}$ \\
\hline \multirow[t]{3}{*}{ b. } & \multirow[t]{3}{*}{ Spermidine } & $\begin{array}{l}1.26 \\
(00)\end{array}$ & $\begin{array}{c}1.52 \\
(+1.20)\end{array}$ & $\begin{array}{c}1.72 \\
(+1.37)\end{array}$ & $\begin{array}{c}0.94 \\
(-0.74)\end{array}$ & $\begin{array}{c}1.92 \\
(+1.52)\end{array}$ & $\begin{array}{c}2.98 \\
(+2.37)\end{array}$ & $\begin{array}{l}\text { Over the control of } \\
\text { normal soil }\end{array}$ \\
\hline & & - & - & - & $(00)$ & $(+2.04)$ & $(+3.17)$ & $\begin{array}{l}\text { Over the control of } \\
\text { sodic soil }\end{array}$ \\
\hline & & - & - & - & - & $(+1.26)$ & $(+1.73)$ & $\begin{array}{l}\text { Over respective } \\
\text { Spermidine conc }\end{array}$ \\
\hline 2. & \multicolumn{7}{|c|}{ CoC 671 (Salt susceptible cultivar) } & \\
\hline \multirow[t]{3}{*}{ a. } & \multirow[t]{3}{*}{ Putrescine } & $\begin{array}{l}1.18 \\
(00)\end{array}$ & $\begin{array}{c}1.52 \\
(+1.28)\end{array}$ & $\begin{array}{c}1.80 \\
(+1.53)\end{array}$ & $\begin{array}{c}0.82 \\
(-0.69)\end{array}$ & $\begin{array}{c}2.46 \\
(+2.08)\end{array}$ & $\begin{array}{c}3.88 \\
(+3.28)\end{array}$ & $\begin{array}{c}\text { Over the control of } \\
\text { normal soil }\end{array}$ \\
\hline & & - & - & - & (00) & $(+3)$ & $(+4.73)$ & $\begin{array}{l}\text { Over the control of } \\
\text { sodic soil }\end{array}$ \\
\hline & & - & - & - & - & $(+1.61)$ & $(+2.16)$ & $\begin{array}{c}\text { Over respective } \\
\text { Putrescine conc. }\end{array}$ \\
\hline \multirow[t]{11}{*}{ b. } & \multirow[t]{3}{*}{ Spermidine } & $\begin{array}{l}1.16 \\
(00)\end{array}$ & $\begin{array}{c}1.46 \\
(+1.25)\end{array}$ & $\begin{array}{c}1.74 \\
(+1.5)\end{array}$ & $\begin{array}{c}0.86 \\
(-0.74)\end{array}$ & $\begin{array}{l}2.32 \\
(+2)\end{array}$ & $\begin{array}{c}3.70 \\
(+3.18)\end{array}$ & $\begin{array}{l}\text { Over the control of } \\
\text { normal soil }\end{array}$ \\
\hline & & - & - & - & $(00)$ & $(+2.69)$ & $(+4.30)$ & $\begin{array}{l}\text { Over the control of } \\
\text { sodic soil }\end{array}$ \\
\hline & & - & - & - & - & $(+1.58)$ & $(+2.12)$ & $\begin{array}{l}\text { Over respective } \\
\text { Spermidine conc }\end{array}$ \\
\hline & Comparison & $\begin{array}{l}\text { S.Em. } \\
\pm\end{array}$ & $\begin{array}{l}\mathrm{CD} \text { at } \\
5 \%\end{array}$ & \multicolumn{2}{|c|}{ Comparison } & $\begin{array}{l}\text { S.Em. } \\
\pm\end{array}$ & $\mathrm{CD}$ at $5 \%$ & \\
\hline & Variety (V) & 0.019 & 0.05 & & $\mathrm{~T} * \mathrm{~L}$ & $0 . \overline{033}$ & 0.09 & \\
\hline & Soil (S) & 0.019 & NS & & $\mathrm{T} * \mathrm{~S}$ & 0.027 & NS & \\
\hline & $\begin{array}{c}\text { Type of } \\
\text { polyamine }(\mathrm{T})\end{array}$ & 0.019 & 0.05 & & $\mathrm{~V} * \mathrm{~S} * \mathrm{~T}$ & 0.038 & 0.11 & \\
\hline & $\begin{array}{c}\text { Conc. of } \\
\text { Polyamines (L) }\end{array}$ & 0.023 & 0.06 & & $\mathrm{~V} * \mathrm{~T} * \mathrm{~L}$ & 0.047 & 0.13 & \\
\hline & $\mathrm{V} * \mathrm{~S}$ & 0.027 & NS & & $\mathrm{S} * \mathrm{~T} * \mathrm{~L}$ & 0.047 & 0.13 & \\
\hline & \multirow[t]{2}{*}{$\mathrm{V} * \mathrm{~T}$} & 0.027 & 0.07 & & $\mathrm{~V} * \mathrm{~S} * \mathrm{~L}$ & 0.047 & 0.13 & \\
\hline & & & & & $\mathbf{V} * \mathbf{S} * \mathbf{T} * \mathbf{L}$ & 0.067 & 0.19 & \\
\hline
\end{tabular}


Table.4 Effect of polyamines on lipid peroxidation in sugarcane leaves grown on normal and sodic soils

\begin{tabular}{|c|c|c|c|c|c|c|c|c|}
\hline \multirow{4}{*}{$\begin{array}{l}\text { Sr. } \\
\text { No. }\end{array}$} & \multirow{4}{*}{$\begin{array}{l}\text { Name of the } \\
\text { cultivar/ } \\
\text { Name of the } \\
\text { polyamine }\end{array}$} & \multicolumn{6}{|c|}{ Lipid peroxidation ( $\eta$ moles of MDA formed $\mathrm{mg}^{-1}$ fr.wt.) } & \multirow{4}{*}{$\begin{array}{c}\text { Fold Increase }(+) / \\
\text { Fold Decrease } \\
(-)\end{array}$} \\
\hline & & \multirow{2}{*}{\multicolumn{3}{|c|}{ Normal soil }} & \multirow{2}{*}{\multicolumn{3}{|c|}{$\begin{array}{l}\text { Sodic soil } \\
\text { f the polvamine (uM) }\end{array}$}} & \\
\hline & & & & entration & & & & \\
\hline & & $\mathbf{0}$ & 100 & 500 & 0 & 100 & 500 & \\
\hline 1. & \multicolumn{7}{|c|}{ CoM 265 (Salt tolerant cultivar) } & \\
\hline a. & Putrescine & $\begin{array}{c}28.03 \\
(00) \\
-\end{array}$ & $\begin{array}{c}28.00 \\
(-0.99) \\
-\end{array}$ & $\begin{array}{c}27.99 \\
(-0.99) \\
-\end{array}$ & $\begin{array}{l}28.03 \\
(1) \\
(00)\end{array}$ & $\begin{array}{c}28.19 \\
(+1.00) \\
(+1.00) \\
(+1.00)\end{array}$ & $\begin{array}{c}28.10 \\
(+1.00) \\
(+1.00) \\
(+1.00)\end{array}$ & $\begin{array}{l}\text { Over the control of } \\
\text { normal soil } \\
\text { Over the control of } \\
\text { sodic soil } \\
\text { Over respective } \\
\text { Putrescine conc. }\end{array}$ \\
\hline \multirow[t]{3}{*}{ b. } & \multirow[t]{3}{*}{ Spermidine } & $\begin{array}{c}28.00 \\
(00)\end{array}$ & $\begin{array}{c}28.16 \\
(+1.00)\end{array}$ & $\begin{array}{c}28.17 \\
(+1.00)\end{array}$ & $\begin{array}{c}28.31 \\
(+1.01)\end{array}$ & $\begin{array}{c}28.16 \\
(+1.00)\end{array}$ & $\begin{array}{l}28.17 \\
(1.00)\end{array}$ & $\begin{array}{l}\text { Over the control of } \\
\text { normal soil }\end{array}$ \\
\hline & & - & - & - & $(00)$ & $(-0.99)$ & $(-0.99)$ & $\begin{array}{c}\text { Over the control of } \\
\text { sodic soil }\end{array}$ \\
\hline & & - & - & - & - & (1) & (1) & $\begin{array}{l}\text { Over respective } \\
\text { Spermidine conc }\end{array}$ \\
\hline 2. & \multicolumn{7}{|c|}{ CoC 671 (Salt susceptible cultivar) } & \\
\hline \multirow[t]{3}{*}{ a. } & \multirow[t]{3}{*}{ Putrescine } & $\begin{array}{c}18.55 \\
(00)\end{array}$ & $\begin{array}{c}18.50 \\
(-0.99)\end{array}$ & $\begin{array}{c}18.48 \\
(-0.99)\end{array}$ & $\begin{array}{c}21.07 \\
(+1.13)\end{array}$ & $\begin{array}{c}19.44 \\
(+1.04)\end{array}$ & $\begin{array}{c}18.88 \\
(+1.01)\end{array}$ & $\begin{array}{l}\text { Over the control of } \\
\text { normal soil }\end{array}$ \\
\hline & & - & - & - & $(00)$ & $(-0.92)$ & $(-0.89)$ & $\begin{array}{l}\text { Over the control of } \\
\text { sodic soil }\end{array}$ \\
\hline & & - & - & - & - & $(+1.05)$ & $(+1.02)$ & $\begin{array}{l}\text { Over respective } \\
\text { Putrescine conc. }\end{array}$ \\
\hline \multirow[t]{11}{*}{ b. } & \multirow[t]{3}{*}{ Spermidine } & $\begin{array}{c}18.41 \\
(00)\end{array}$ & $\begin{array}{c}18.40 \\
(+0.99)\end{array}$ & $\begin{array}{c}18.38 \\
(-0.99)\end{array}$ & $\begin{array}{l}21.13 \\
(1.14)\end{array}$ & $\begin{array}{c}19.15 \\
(+1.04)\end{array}$ & $\begin{array}{c}18.66 \\
(+1.01)\end{array}$ & $\begin{array}{c}\text { Over the control of } \\
\text { normal soil }\end{array}$ \\
\hline & & - & - & - & (00) & $(-0.90)$ & $(-0.88)$ & $\begin{array}{l}\text { Over the control of } \\
\text { sodic soil }\end{array}$ \\
\hline & & - & - & - & - & $(+1.04)$ & $(+1.02)$ & $\begin{array}{l}\text { Over respective } \\
\text { Spermidine conc }\end{array}$ \\
\hline & Comparison & $\begin{array}{l}\text { S.Em. } \\
\pm\end{array}$ & $\begin{array}{l}\mathrm{CD} \text { at } \\
5 \%\end{array}$ & & Comparison & $\begin{array}{l}\text { S.Em. } \\
\pm\end{array}$ & $\begin{array}{l}\mathrm{CD} \text { at } \\
5 \%\end{array}$ & \\
\hline & Variety (V) & 0.098 & 0.28 & & $\mathrm{~T} * \mathrm{~L}$ & $0 . \overline{181}$ & 0.51 & \\
\hline & Soil (S) & 0.100 & 0.28 & & $\mathrm{~T} * \mathrm{~S}$ & 0.145 & 0.55 & \\
\hline & $\begin{array}{c}\text { Type of } \\
\text { polyamine }(\mathrm{T})\end{array}$ & 0.100 & 0.28 & & $\mathrm{~V} * \mathrm{~S} * \mathrm{~T}$ & 0.197 & 0.56 & \\
\hline & $\begin{array}{c}\text { Conc. of } \\
\text { Polyamines (L) }\end{array}$ & 0.124 & 0.35 & & $\mathrm{~V} * \mathrm{~T} * \mathrm{~L}$ & 0.242 & 0.68 & \\
\hline & $\mathrm{V} * \mathrm{~S}$ & 0.597 & 1.69 & & $\mathrm{~S} * \mathrm{~T} * \mathrm{~L}$ & 0.270 & 0.76 & \\
\hline & $\mathrm{V} * \mathrm{~T}$ & 0.597 & 1.69 & & $\mathrm{~V} * \mathrm{~S} * \mathrm{~L}$ & 0.242 & 0.68 & \\
\hline & & & & & $\mathbf{V} * \mathbf{S} * \mathbf{T} * \mathbf{L}$ & 0.342 & NS & \\
\hline
\end{tabular}


Table.5 Effect of polyamines on reducing sugars in sugarcane leaves grown on normal and sodic soils

\begin{tabular}{|c|c|c|c|c|c|c|c|c|}
\hline \multirow{4}{*}{$\begin{array}{l}\text { Sr. } \\
\text { No. }\end{array}$} & \multirow{4}{*}{$\begin{array}{l}\text { Name of the } \\
\text { cultivar/ } \\
\text { Name of the } \\
\text { polyamine }\end{array}$} & \multicolumn{6}{|c|}{ Reducing sugars $\left(\mathrm{mg} \mathrm{g}^{-1} \mathrm{DW}\right)$} & \multirow{4}{*}{$\begin{array}{c}\text { Fold Increase }(+) / \\
\text { Fold Decrease } \\
(-)\end{array}$} \\
\hline & & \multicolumn{3}{|c|}{ Normal soil } & \multirow{2}{*}{\multicolumn{3}{|c|}{$\begin{array}{r}\text { Sodic soil } \\
\text { of the polyamine }(\mu \mathrm{M})\end{array}$}} & \\
\hline & & \multicolumn{3}{|c|}{ Concentration of the polyamine $(\mu \mathrm{M})$} & & & & \\
\hline & & $\mathbf{0}$ & 100 & 500 & 0 & 100 & 500 & \\
\hline 1. & \multicolumn{7}{|c|}{ CoM 265 (Salt tolerant cultivar) } & \\
\hline a. & Putrescine & $\begin{array}{l}3.33 \\
(00)\end{array}$ & $\begin{array}{c}3.48 \\
(+1.04) \\
-\end{array}$ & $\begin{array}{c}3.66 \\
(+1.09) \\
-\end{array}$ & $\begin{array}{c}2.89 \\
(-0.86) \\
(00)\end{array}$ & $\begin{array}{c}3.14 \\
(-0.94) \\
(+1.08) \\
(-0.90)\end{array}$ & $\begin{array}{c}3.52 \\
(+1.05) \\
(+1.21) \\
(-0.96)\end{array}$ & $\begin{array}{c}\text { Over the control of } \\
\text { normal soil } \\
\text { Over the control of sodic } \\
\text { soil } \\
\text { Over respective } \\
\text { Putrescine conc. }\end{array}$ \\
\hline \multirow[t]{3}{*}{ b. } & \multirow[t]{3}{*}{ Spermidine } & $\begin{array}{l}3.00 \\
(00)\end{array}$ & $\begin{array}{c}3.15 \\
(+1.05)\end{array}$ & $\begin{array}{c}3.27 \\
(+1.09)\end{array}$ & $\begin{array}{c}2.90 \\
(+0.96)\end{array}$ & $\begin{array}{c}3.01 \\
(+1.00)\end{array}$ & $\begin{array}{c}3.22 \\
(+1.07)\end{array}$ & $\begin{array}{c}\text { Over the control of } \\
\text { normal soil }\end{array}$ \\
\hline & & - & - & - & (00) & $(+1.03)$ & $(+1.10)$ & $\begin{array}{c}\text { Over the control of sodic } \\
\text { soil }\end{array}$ \\
\hline & & - & - & - & - & $(-0.96)$ & $(-0.98)$ & $\begin{array}{l}\text { Over respective } \\
\text { Spermidine conc }\end{array}$ \\
\hline 2. & \multicolumn{7}{|c|}{ CoC 671 (Salt susceptible cultivar) } & \\
\hline \multirow[t]{3}{*}{ a. } & \multirow[t]{3}{*}{ Putrescine } & $\begin{array}{l}2.03 \\
(00)\end{array}$ & $\begin{array}{c}2.26 \\
(+1.11)\end{array}$ & $\begin{array}{c}2.60 \\
(+1.28)\end{array}$ & $\begin{array}{c}1.78 \\
(+0.87)\end{array}$ & $\begin{array}{c}2.57 \\
(+1.26)\end{array}$ & $\begin{array}{c}3.26 \\
(+1.60)\end{array}$ & $\begin{array}{l}\text { Over the control of } \\
\text { normal soil }\end{array}$ \\
\hline & & - & - & - & (00) & $(+1.44)$ & $(+1.83)$ & $\begin{array}{c}\text { Over the control of sodic } \\
\text { soil }\end{array}$ \\
\hline & & - & - & - & - & $(+1.13)$ & $(+1.25)$ & $\begin{array}{l}\text { Over respective } \\
\text { Putrescine conc. }\end{array}$ \\
\hline \multirow[t]{11}{*}{ b. } & \multirow[t]{3}{*}{ Spermidine } & $\begin{array}{l}2.00 \\
(00)\end{array}$ & $\begin{array}{c}2.15 \\
(+1.08)\end{array}$ & $\begin{array}{c}2.29 \\
(+1.15)\end{array}$ & $\begin{array}{c}1.80 \\
(+0.9)\end{array}$ & $\begin{array}{c}2.14 \\
(+1.07)\end{array}$ & $\begin{array}{c}2.45 \\
(+1.23)\end{array}$ & $\begin{array}{c}\text { Over the control of } \\
\text { normal soil }\end{array}$ \\
\hline & & - & - & - & (00) & $(+1.18)$ & $(+1.36)$ & $\begin{array}{c}\text { Over the control of sodic } \\
\text { soil }\end{array}$ \\
\hline & & - & - & - & - & $(-0.100)$ & $(+1.06)$ & $\begin{array}{l}\text { Over respective } \\
\text { Spermidine conc }\end{array}$ \\
\hline & Comparison & $\begin{array}{l}\text { S.Em. } \\
\pm\end{array}$ & $\begin{array}{l}\mathrm{CD} \text { at } \\
5 \%\end{array}$ & & Comparison & S.Em. \pm & $\begin{array}{l}\mathrm{CD} \text { at } \\
5 \%\end{array}$ & \\
\hline & Variety (V) & $0 . \overline{0} 66$ & 0.017 & & $\mathrm{~T} * \mathrm{~L}$ & 0.010 & 0.030 & \\
\hline & Soil (S) & 0.006 & 0.017 & & $\mathrm{~T} * \mathrm{~S}$ & 0.008 & 0.024 & \\
\hline & $\begin{array}{c}\text { Type of } \\
\text { polyamine }(\mathrm{T})\end{array}$ & 0.006 & 0.017 & & $\mathrm{~V} * \mathrm{~S} * \mathrm{~T}$ & 0.012 & 0.034 & \\
\hline & $\begin{array}{c}\text { Conc. of } \\
\text { Polyamines (L) }\end{array}$ & 0.007 & 0.021 & & $\mathrm{~V} * \mathrm{~T} * \mathrm{~L}$ & 0.015 & 0.042 & \\
\hline & $\mathrm{V} * \mathrm{~S}$ & 0.008 & 0.024 & & $\mathrm{~S} * \mathrm{~T} * \mathrm{~L}$ & 0.015 & 0.042 & \\
\hline & $\mathrm{V}^{*} \mathrm{~T}$ & 0.008 & 0.024 & & $\mathrm{~V} * \mathrm{~S} * \mathrm{~L}$ & 0.015 & 0.042 & \\
\hline & & & & & $\mathbf{V} * \mathbf{S} * \mathbf{T} * \mathbf{L}$ & 0.021 & 0.060 & \\
\hline
\end{tabular}


Table.6 Effect of polyamines on non-reducing sugars in sugarcane leaves grown on normal and sodic soils

\begin{tabular}{|c|c|c|c|c|c|c|c|c|}
\hline \multirow{4}{*}{$\begin{array}{l}\text { Sr. } \\
\text { No. }\end{array}$} & \multirow{4}{*}{$\begin{array}{l}\text { Name of the } \\
\text { cultivar/ } \\
\text { Name of the } \\
\text { polyamine }\end{array}$} & \multicolumn{6}{|c|}{ Non-Reducing sugars ( $\left.\mathrm{mg} \mathrm{g}^{-1} \mathrm{DW}\right)$} & \multirow{4}{*}{$\begin{array}{c}\text { Fold Increase }(+) / \\
\text { Fold Decrease } \\
(-)\end{array}$} \\
\hline & & \multicolumn{3}{|c|}{ Normal soil } & \multirow{2}{*}{\multicolumn{3}{|c|}{$\begin{array}{r}\text { Sodic soil } \\
\text { mine }(\mu \mathrm{M})\end{array}$}} & \\
\hline & & & Conc & ntration & & & & \\
\hline & & $\mathbf{0}$ & 100 & 500 & 0 & 100 & 500 & \\
\hline 1. & \multicolumn{7}{|c|}{ CoM 265 (Salt tolerant cultivar) } & \\
\hline a. & Putrescine & $\begin{array}{l}4.25 \\
(00) \\
-\end{array}$ & $\begin{array}{c}4.39 \\
(+1.03) \\
-\end{array}$ & $\begin{array}{c}4.56 \\
(+1.07) \\
-\end{array}$ & $\begin{array}{c}3.55 \\
(-0.83) \\
\\
(00) \\
-\end{array}$ & $\begin{array}{c}3.80 \\
(-0.89) \\
(+1.07) \\
(-0.87)\end{array}$ & $\begin{array}{c}4.18 \\
(+0.98) \\
(+1.17) \\
(-0.91)\end{array}$ & $\begin{array}{l}\text { Over the control of } \\
\text { normal soil } \\
\text { Over the control of } \\
\text { sodic soil } \\
\text { Over respective } \\
\text { Putrescine conc. }\end{array}$ \\
\hline b. & Spermidine & $\begin{array}{c}4.26 \\
(00) \\
-\end{array}$ & $\begin{array}{c}4.41 \\
(+1.03) \\
- \\
-\end{array}$ & $\begin{array}{c}4.53 \\
(+1.06) \\
- \\
-\end{array}$ & $\begin{array}{c}3.56 \\
(-0.84) \\
(00) \\
-\end{array}$ & $\begin{array}{c}3.67 \\
(-0.86) \\
(+1.03) \\
(-0.83)\end{array}$ & $\begin{array}{c}3.88 \\
(+0.91) \\
(+1.08) \\
(-0.85)\end{array}$ & $\begin{array}{l}\begin{array}{l}\text { Over the control of } \\
\text { normal soil }\end{array} \\
\text { Over the control of } \\
\text { sodic soil } \\
\text { Over respective } \\
\text { Spermidine conc } \\
\end{array}$ \\
\hline 2. & \multicolumn{7}{|c|}{ CoC 671 (Salt susceptible cultivar) } & \\
\hline a. & Putrescine & $\begin{array}{c}3.36 \\
(00) \\
-\end{array}$ & $\begin{array}{c}3.59 \\
(+1.06)\end{array}$ & $\begin{array}{c}3.93 \\
(+1.16) \\
-\end{array}$ & $\begin{array}{c}1.89 \\
(-0.56) \\
(00)\end{array}$ & $\begin{array}{c}2.68 \\
(-0.79) \\
(+1.41) \\
(-0.91)\end{array}$ & $\begin{array}{c}3.37 \\
(+1.00) \\
(+1.78) \\
(-0.85)\end{array}$ & $\begin{array}{l}\text { Over the control of } \\
\text { normal soil } \\
\text { Over the control of } \\
\text { sodic soil } \\
\text { Over respective } \\
\text { Putrescine conc. }\end{array}$ \\
\hline \multirow[t]{9}{*}{ b. } & Spermidine & $\begin{array}{c}3.33 \\
(00) \\
-\end{array}$ & $\begin{array}{c}3.48 \\
(+1.04) \\
-\end{array}$ & $\begin{array}{c}3.62 \\
(+1.08) \\
- \\
-\end{array}$ & $\begin{array}{c}1.80 \\
(-0.54) \\
(00) \\
-\end{array}$ & $\begin{array}{c}2.14 \\
(-0.64) \\
(+1.18) \\
(-0.61)\end{array}$ & $\begin{array}{c}2.45 \\
(-0.73) \\
(+1.36) \\
(-0.67)\end{array}$ & $\begin{array}{l}\text { Over the control of } \\
\text { normal soil } \\
\text { Over the control of } \\
\text { sodic soil } \\
\text { Over respective } \\
\text { Spermidine conc }\end{array}$ \\
\hline & Comparison & $\begin{array}{l}\text { S.Em. } \\
\pm\end{array}$ & $\begin{array}{l}\mathrm{CD} \text { at } \\
5 \%\end{array}$ & & Comparison & $\begin{array}{l}\text { S.Em. } \\
\pm\end{array}$ & $\begin{array}{l}\mathrm{CD} \text { at } \\
5 \%\end{array}$ & \\
\hline & Variety (V) & 0.007 & 0.021 & & $\mathrm{~T} * \mathrm{~L}$ & $0 . \overline{0} 13$ & 0.038 & \\
\hline & Soil (S) & 0.007 & 0.021 & & $\mathrm{~T} * \mathrm{~S}$ & 0.010 & 0.030 & \\
\hline & $\begin{array}{c}\text { Type of } \\
\text { polyamine }(\mathrm{T})\end{array}$ & 0.007 & 0.021 & & $\mathrm{~V} * \mathrm{~S} * \mathrm{~T}$ & 0.015 & 0.043 & \\
\hline & $\begin{array}{c}\text { Conc. of } \\
\text { Polyamines (L) }\end{array}$ & 0.009 & 0.026 & & $\mathrm{~V} * \mathrm{~T} * \mathrm{~L}$ & 0.018 & 0.053 & \\
\hline & $\mathrm{V} * \mathrm{~S}$ & 0.010 & 0.030 & & $\mathrm{~S} * \mathrm{~T} * \mathrm{~L}$ & 0.018 & 0.053 & \\
\hline & $\mathrm{V} * \mathrm{~T}$ & 0.010 & 0.030 & & $\mathrm{~V} * \mathrm{~S} * \mathrm{~L}$ & 0.018 & 0.053 & \\
\hline & & & & & $\mathbf{V} * \mathbf{S} * \mathbf{T} * \mathbf{L}$ & 0.026 & 0.075 & \\
\hline
\end{tabular}


Table.7 Effect of polyamines on total sugars in sugarcane leaves grown on normal and sodic soils

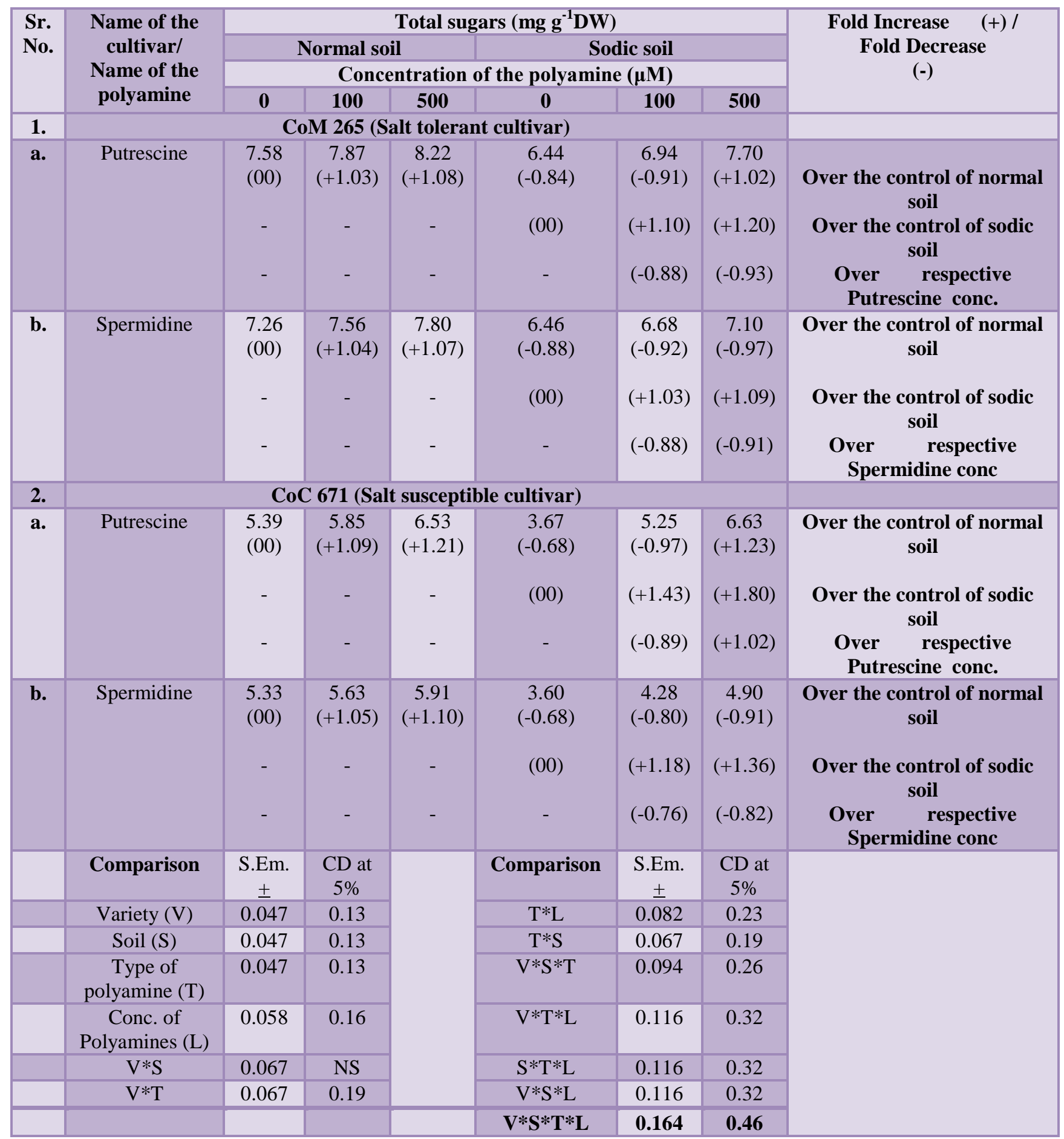

The lipid peroxidation of the variety CoC 671 at concentration of putrescine and spermidine was 21.07 moles of MDA formed $\mathrm{mg}^{-1}$ fr.wt.
$(0 \mu \mathrm{M}), 19.44 \eta$ moles of MDA formed $\mathrm{mg}^{-1}$ fr.wt. $(100 \mu \mathrm{M}), 18.88 \eta$ moles of MDA formed $\mathrm{mg}^{-1}$ fr.wt. $(500 \mu \mathrm{M})$ and $21.13 \eta$ moles of 
MDA formed $\mathrm{mg}^{-1}$ fr.wt. $(0 \mu \mathrm{M}), 19.15 \eta$ moles of MDA formed $\mathrm{mg}^{-1}$ fr.wt. $(100 \mu \mathrm{M})$, $18.66 \eta$ moles of MDA formed $\mathrm{mg}^{-1}$ fr.wt. $(500 \mu \mathrm{M})$ in sodic soil.

Hosseini et al., (2010) observed that MDA level increased gradually in two varieties of sugarcane by $\mathrm{NaCl}$ stress. Similar trend was observed in the present study due to sodicity. Similar trend was also reported by Pagariya et al., (2012) in sugarcane leaves. The membrane damage is caused by lipid peroxidation resulting from the uncontrolled ROS production (Rodriquez-Rosales et al., 1999). Hala et al., (2005) reported that salt stress decreased the PUT level in two wheat cultivars with increase in salinity level. However, Spd and Spm were increased with increase in the salinity levels in two wheat cultivars. Erdei et al., (1996) indicated that Spd and Spm accumulation is one of the adaptive responses to salt stress. In the present investigation application PAs resulted in lowering lipid peroxidation in sugarcane cultivars.

\section{Reducing sugar}

The reducing sugar in the leaves of two sugarcane varieties viz., CoM 0265 and CoC 671 were decreased when grown in sodic soil (Table 5). The effect of polyamines on reducing sugars in sugarcane grown under normal and sodic soil the resulted in increased in reducing sugar with increase in the polyamine concentration in both on the cultivar (Table 5).

The reducing sugar of the variety CoM 0265 at concentration of putrescine and spermidine was $3.33 \mathrm{mg} \mathrm{g}^{-1}(0 \mu \mathrm{M}), 3.48 \mathrm{mg} \mathrm{g}^{-1}(100 \mu \mathrm{M})$, $3.66 \mathrm{mg} \mathrm{g}^{-1}(500 \mu \mathrm{M})$ and $3.00 \mathrm{mg} \mathrm{g}^{-1}(0 \mu \mathrm{M})$, $3.15 \mathrm{mg} \mathrm{g}^{-1}(100 \mu \mathrm{M}), 3.27 \mathrm{mg} \mathrm{g}^{-1}(500 \mu \mathrm{M})$ in normal soil. The reducing sugar of the variety CoM 0265 at concentration of putrescine and spermidine was $2.89 \mathrm{mg} \mathrm{g}^{-1}(0 \mu \mathrm{M}), 3.14 \mathrm{mg} \mathrm{g}^{-1}$ $(100 \mu \mathrm{M}), 3.52 \mathrm{mg} \mathrm{g}^{-1}(500 \mu \mathrm{M})$ and $2.90 \mathrm{mg} \mathrm{g}^{-1}$
$(0 \mu \mathrm{M}), \quad 3.01 \mathrm{mg}^{-1} \quad(100 \mu \mathrm{M}), \quad 3.22 \mathrm{mg} \mathrm{g}^{-1}$ $(500 \mu \mathrm{M})$ in sodic soil. The reducing sugar of the variety $\mathrm{CoC} 671$ at concentration of putrescine and spermidine was $2.03 \mathrm{mg} \mathrm{g}^{-1}$ $(0 \mu \mathrm{M}), \quad 2.26 \mathrm{mg} \mathrm{g}^{-1}(100 \mu \mathrm{M}), \quad 2.60 \mathrm{mg} \mathrm{g}^{-1}$ $(500 \mu \mathrm{M})$ and $2.00 \mathrm{mg} \mathrm{g}^{-1}(0 \mu \mathrm{M}), 2.15 \mathrm{mg} \mathrm{g}^{-1}$ $(100 \mu \mathrm{M}), 2.29 \mathrm{mg} \mathrm{g}^{-1}(500 \mu \mathrm{M})$ in normal soil. The reducing sugar of the variety $\mathrm{CoC} 671$ at concentration of putrescine and spermidine was $1.78 \mathrm{mg} \mathrm{g}^{-1}(0 \mu \mathrm{M}), 2.57 \mathrm{mg} \mathrm{g}^{-1}(100 \mu \mathrm{M})$, $3.26 \mathrm{mg} \mathrm{g}^{-1}(500 \mu \mathrm{M})$ and $1.80 \mathrm{mg} \mathrm{g}^{-1}(0 \mu \mathrm{M})$, $2.14 \mathrm{mg} \mathrm{g}^{-1}(100 \mu \mathrm{M}), 2.45 \mathrm{mg} \mathrm{g}^{-1}(500 \mu \mathrm{M})$ in sodic soil.

\section{Non reducing sugar}

The sugarcane varieties, CoM 0265 and CoC 671 grown in sodic soil caused the reduction in the reducing sugars (Table 6). The effect of polyamines on non reducing sugars of sugarcane leaves grown under normal and sodic soil resulted increased with increase in the polyamine concentration in both on the cultivar (Table 6). The non reducing sugar in the variety CoM 0265 at concentration of putrescine and spermidine was $4.25 \mathrm{mg} \mathrm{g}^{-1}$ $(0 \mu \mathrm{M}), \quad 4.39 \mathrm{mg} \mathrm{g}^{-1}(100 \mu \mathrm{M}), \quad 4.56 \mathrm{mg} \mathrm{g}^{-1}$ $(500 \mu \mathrm{M})$ and $4.26 \mathrm{mg} \mathrm{g}^{-1}(0 \mu \mathrm{M}), 4.41 \mathrm{mg} \mathrm{g}^{-1}$ $(100 \mu \mathrm{M}), 4.53 \mathrm{mg} \mathrm{g}^{-1}(500 \mu \mathrm{M})$ in normal soil. The non-reducing sugar of the variety CoM 0265 at concentration of putrescine and spermidine was $3.55 \mathrm{mg} \mathrm{g}^{-1}(0 \mu \mathrm{M}), 3.80 \mathrm{mg} \mathrm{g}^{-1}$ $(100 \mu \mathrm{M}), 4.18 \mathrm{mg} \mathrm{g}^{-1}(500 \mu \mathrm{M})$ and $3.56 \mathrm{mg} \mathrm{g}^{-1}$ $(0 \mu \mathrm{M}), \quad 3.67 \mathrm{mg} \mathrm{g}^{-1}(100 \mu \mathrm{M}), \quad 3.88 \mathrm{mg} \mathrm{g}^{-1}$ $(500 \mu \mathrm{M})$ in sodic soil. The non-reducing sugar of the variety $\mathrm{CoC} 671$ at concentration of putrescine and spermidine was $3.36 \mathrm{mg} \mathrm{g}^{-1}$ $(0 \mu \mathrm{M}), \quad 3.59 \mathrm{mg} \mathrm{g}^{-1}(100 \mu \mathrm{M}), 3.93 \mathrm{mg} \mathrm{g}^{-1}$ $(500 \mu \mathrm{M})$ and $3.33 \mathrm{mg} \mathrm{g}^{-1}(0 \mu \mathrm{M}), 3.48 \mathrm{mg} \mathrm{g}^{-1}$ $(100 \mu \mathrm{M}), 3.62 \mathrm{mg} \mathrm{g}^{-1}(500 \mu \mathrm{M})$ in normal soil. The non-reducing sugar of the variety $\mathrm{CoC}$ 671 at concentration of putrescine and spermidine was $1.89 \mathrm{mg} \mathrm{g}^{-1}(0 \mu \mathrm{M}), 2.68 \mathrm{mg} \mathrm{g}^{-1}$ $(100 \mu \mathrm{M}), 3.37 \mathrm{mg} \mathrm{g}^{-1}(500 \mu \mathrm{M})$ and $1.80 \mathrm{mg} \mathrm{g}^{-1}$ $(0 \mu \mathrm{M}), \quad 2.14 \mathrm{mg} \mathrm{g}^{-1}(100 \mu \mathrm{M}), 2.45 \mathrm{mg} \mathrm{g}^{-1}$ $(500 \mu \mathrm{M})$ in sodic soil. 


\section{Total sugar}

The total sugar was reduced in sugarcane varieties $\mathrm{CoM} 0265$ and $\mathrm{CoC} 671$ when grown in the sodic soil (Table 7). However the application of polyamines on sugarcane plant grown under normal and sodic soil resulted increased in total sugar with increase in the polyamine concentration in both on the cultivar (Table 7). The total sugar of the variety $\mathrm{CoM} 0265$ at concentration of putrescine and spermidine was $7.58 \mathrm{mg} \mathrm{g}^{-1}$ $(0 \mu \mathrm{M}), \quad 7.87 \mathrm{mg} \mathrm{g}^{-1}(100 \mu \mathrm{M}), 8.22 \mathrm{mg} \mathrm{g}^{-1}$ $(500 \mu \mathrm{M})$ and $7.26 \mathrm{mg} \mathrm{g}^{-1}(0 \mu \mathrm{M}), 7.56 \mathrm{mg} \mathrm{g}^{-1}$ $(100 \mu \mathrm{M}), 7.80 \mathrm{mg} \mathrm{g}^{-1}(500 \mu \mathrm{M})$ in normal soil. The total sugar of the variety CoM 0265 at concentration of putrescine and spermidine was $6.44 \mathrm{mg} \mathrm{g}^{-1}(0 \mu \mathrm{M}), 6.94 \mathrm{mg} \mathrm{g}^{-1}(100 \mu \mathrm{M})$, $7.70 \mathrm{mg} \mathrm{g}^{-1}(500 \mu \mathrm{M})$ and $6.46 \mathrm{mg} \mathrm{g}^{-1}(0 \mu \mathrm{M})$, $6.68 \mathrm{mg} \mathrm{g}^{-1}(100 \mu \mathrm{M}), 7.20 \mathrm{mg} \mathrm{g}^{-1}(500 \mu \mathrm{M})$ in sodic soil. The total sugar of the variety $\mathrm{CoC}$ 671 at concentration of putrescine and spermidine was $5.39 \mathrm{mg} \mathrm{g}^{-1}(0 \mu \mathrm{M}), 5.85 \mathrm{mg} \mathrm{g}^{-1}$ $(100 \mu \mathrm{M}), 6.53 \mathrm{mg} \mathrm{g}^{-1}(500 \mu \mathrm{M})$ and $5.33 \mathrm{mg} \mathrm{g}^{-1}$ $(0 \mu \mathrm{M}), \quad 5.63 \mathrm{mg} \mathrm{g}^{-1}(100 \mu \mathrm{M}), \quad 5.91 \mathrm{mg} \mathrm{g}^{-1}$ $(500 \mu \mathrm{M})$ in normal soil. The total sugar of the variety $\mathrm{CoC} 671$ at concentration of putrescine and spermidine was $3.67 \mathrm{mg} \mathrm{g}^{-1}(0 \mu \mathrm{M})$, $5.25 \mathrm{mg} \mathrm{g}^{-1}(100 \mu \mathrm{M}), 6.63 \mathrm{mg} \mathrm{g}^{-1}(500 \mu \mathrm{M})$ and $3.60 \mathrm{mg} \mathrm{g}^{-1}(0 \mu \mathrm{M}), \quad 4.28 \mathrm{mg} \mathrm{g}^{-1}(100 \mu \mathrm{M})$, $4.90 \mathrm{mg} \mathrm{g}^{-1}(500 \mu \mathrm{M})$ in sodic soil.

In the present study the reduction in sugars in sugarcane leaves when grown under sodic soil may be due to losses in the photosynthetic activity with a drop in the net formation of carbohydrates in general (Trivedi et al., 2004 and Gadallah, 1999). In cotton, foliar applications with Put increased total sugars under different levels of salinity compared to control plants (Ahmed et al., 2013). Similar trend was observed in the present investigation. The increase in sugar in the present study by the application of PAs may have role act as osmoprotectants, regulating the cell osmotic status, protecting the membranes, and contributing to the scavenging of free radicals in plant cells. (Leopold et al., 1994).

\section{References}

Ahmed, A. H. Darwish, E. Hamoda, S. A. F. and Alobaidy, M. G. 2013. Effect putrescine and humic acid on growth, yield and chemical composition of cotton plants growth under saline soil condition American-Eurasian J. Agric, and Environ.Sci. 13 (4): 479-497.

Alcazar, R., Altabella, T., Marco, F., Bortolotti, C., Reymond, M., Knocz, C., Carrasco, P. and Tiburcio, A.F. 2010. Polyamines molecules with regulatory functions in plant abiotic stress tolerance. Planta. 231: 1237-49.

Alcázar, R., Altabella, T., Marco, F., Bortolotti, C., Reymond, M., Koncz, C., Carrasco, P. and Tiburcio, A. F. 2006. Involvement of polyamines in plant response to abiotic stress. Biotech. Letters. 28: 1867-1876.

Alet, A. I., Sánchez, D. H., Cuevas, J.C., Marina. M., Carrasco, P. Altabella, T. Tiburcio, A. F. and Ruiz, O. A. 2012.New insights into the role of spermine in Arabidopsis thaliana under long-term salt stress. Plant Sci. 182: 94100.

Arnon, D. I. 1949. Copper enzymes in isolated chloroplasts, polphennoloxidase in Beta vulgaris L. Plant Physiol. 24(1): 1-15.

Anonymous, 2018. Sugarcane: Area, Production and yield during 2015-16 and 2016-17 in major producing states along with coverage under irrigation. "Agricultural statistics at Glance 2017". Ed by Govt. of India, Ministry of Agriculture and farmers welfare, Dept. of Agril. corp. and farmers welfare, Directorate of Economic and statistics.: Pp. 13.

Chattopadhayay, M. K., Tiwari, B. S., Chattopadhyay, G., Bose, A., Sengupta, D. N. and Ghosh, B. 2002. Protective role of exogenous polyamines on 
salinity-stressed rice Oryza sativa plants. Physiol. Plant 116: 192-199.

Duan, J., Li, J., Guo, S. and Kang, Y. 2008. Exogenous spermidine affects polyamine metabolism in salinitystressed Cucumis sativus roots and enhances short-term salinity tolerance. J. Plant Physiol. 165: 1620-1635.

Erdei, L. Z., Szegletes, K. Barabas. and A. Pestenacz. 1996. Responses in polyamine titer under osmotic and salt stress in sorghum and maize seedling. J. Plant Physiol. 147: 599-603.

Gadallah, M.A.A. 1999. Effects of kinetin on growth, grain yield and some mineral elements in wheat plants growing under excess salinity and oxygen deficienc. Plant Growth Regul. 27: 63-71.

Gill, S.S. and Tuteja, N. 2010.Polyamines and abiotic stress tolerance in plants.Plant Signal Behav. 51: 26-33.

Hala, M.S., EL-Bassiouny and Bekheta, M. A. 2005.The effect of salt stress lipid peroxidation of two wheat cultivars. Inter. J. Agril. Biol. 1560: 363-368.

Heath, R. L. and Packer, L. 1968. Photooxidation in isolated chloroplast, I. kinetics and stoichometry of fatty acid peroxidation. Arch. Biochem. Bio. Phy. 1125: 189- 198.

Hosseini, T., Shekari, F. and Ghorbanli, M. 2010.Effect of salt stress on ion content, proline and antioxidative enzymes of two safflower cultivars Carthamus tinctorius L. J. Food Agric. Environ. 8(2): 10801086.

Kusano, T., Berberich, T., Tateda, C. and Takahashi, Y. 2008. Polyamines: essential factors for growth and survival. Planta 228: 367-381.

Leopold, A.C., Sun, W.C. and Bernal-Lugo, L. 1994. The glassy state in seeds: Analysis and function. Seed Sci. Res. 4: 267-274.

Munns, R. and Termaat, A. 1986. Whole-plant response to salinity. Aust. J. Plant Physiol. 13:143-162.

Nelson, N. 1944.A photometric adaptation of the Somogyi method for the determination of glucose. J. Biol. Chem. 15: 375-380.

Pagariya, M.C., Devarumatha, R.M. and Kawara, P.G. 2012. Biochemical characterization and identification of differentially expressed candidate genes in salt stressed sugarcane. Plant Sci. 184 $: 1-13$

Pang, X.M., Zhang, Z.Y., Wen, X.P., Yusuke, Ban.and Takaya, Moriguchi. 2007. Polyamines, all-purpose players in response to environment stress in plant. Plant stress. Global Sci. Books. 3058572.

Rodriguez-Rosales, M. P., Kerbeb, L., Bueno, P. and Donaire, J. P.1999.Changes induced by $\mathrm{NaCl}$ in lipid content and composition, lipoxygenase, plasma membrane $\mathrm{H}^{+}$ATPase and antioxidant enzyme activities of tomato Lycopersicon esculentum. Millcalli. Plant Sci. 143:143-150.

Wahid, A., Rao, A, and Rasul, E. 1997. Identification of salt tolerance traits in sugarcane lines. Field Crops Res. 54: 917.

Wang, X., Shi, G., Xu, Q. and Hu, J. 2007.Exogenous polyamines enhance copper tolerance of Nymphoides peltatum. J. Plant Physiol. 164: 10621070.

Zhang, R. H., Li, J., Guo, S. R. and Tezuka, T. 2009. Effects of exogenous putrescine on gas exchange characteristics and chlorophyll fluorescence of $\mathrm{NaCl}$ stressed cucumber seedlings. Photosynth Res 100: 155-162.

\section{How to cite this article:}

Chougule, P. S., J. K. Kharat, A. A. Kale, R. M. Naik, A. S. Jadhav and Kute, N. S. 2019. Effect of Polyamine on Physio-Biochemical Traits in Sugarcane Grown under Sodic Soil. Int.J.Curr.Microbiol.App.Sci. 8(12): 1187-1201. doi: https://doi.org/10.20546/ijcmas.2019.812.147 